\title{
Application of qPCR assays based on haloacids transporter gene dehp2 for discrimination of Burkholderia and Paraburkholderia
}

\author{
Xianbin Su${ }^{1 *+} \mathbb{D}, \mathrm{Yi} \mathrm{Shi}^{{ }^{+\dagger}}$, Ruihong $\mathrm{Li}^{2+}$, Zhao-Ning $\mathrm{Lu}^{1+}$, Xin Zou' ${ }^{1}$ Jiao-Xiang $\mathrm{Wu}^{1}$ and Ze-Guang Han ${ }^{1}$
}

\begin{abstract}
Background: A major facilitator superfamily transporter Dehp2 was recently shown to be playing an important role in transport and biodegradation of haloacids in Paraburkholderia caribensis MBA4, and Dehp2 is phylogenetically conserved in Burkholderia sensu lato.

Results: We designed both Burkholderia sensu stricto-specific and Paraburkholderia-specific qPCR assays based on dehp2 and $16 \mathrm{~S}$ rRNA, and validated the qPCR assays in 12 bacterial strains. The qPCR assays could detect single species of Burkholderia sensu stricto or Paraburkholderia with high sensitivity and discriminate them in mixtures with high specificity over a wide dynamic range of relative concentrations. At relatively lower cost compared with sequencing-based approach, the GPCR assays will facilitate discrimination of Burkholderia sensu stricto and Paraburkholderia in a large number of samples.

Conclusions: For the first time, we report the utilization of a haloacids transporter gene for discriminative purpose in Burkholderia sensu lato. This enables not only quick decision on proper handling of putative pathogenic samples in Burkholderia sensu stricto group but also future exploitation of relevant species in Paraburkholderia group for haloacids biodegradation purposes.
\end{abstract}

Keywords: Burkholderia, Paraburkholderia, Haloacids transporter, Dehp2, qPCR, Pathogenicity

\section{Background}

Burkholderia sensu lato includes versatile members that have dramatic different living-styles and occupy diverse ecological niches [1-6]. The most infamous species are Burkholderia pseudomallei and Burkholderia mallei which cause melioidosis and glanders in animals and humans [7-10]. Besides that, Burkholderia cepacia complex (Bcc) includes many closely-related opportunistic pathogens such as Burkholderia cenocepacia and Burkholderia multivorans [11-14]. There are also many species isolated from the environment with biotechnological

\footnotetext{
* Correspondence: xbsu@sjtu.edu.cn

${ }^{+}$Xianbin Su, Yi Shi, Ruihong Li and Zhao-Ning Lu contributed equally to this work.

${ }^{1}$ Key Laboratory of Systems Biomedicine (Ministry of Education), Shanghai Center for Systems Biomedicine, Shanghai Jiao Tong University, Shanghai, China

Full list of author information is available at the end of the article
}

application potentials, such as plant growth promotion, antibiotics production, and biodegradation of pollutants $[4,5,15-17]$, which are generally defined as "plant-beneficial-environmental (PBE) cluster" [18-20]. Phylogenetic analysis based on single gene such as $16 \mathrm{~S}$ rRNA, recA, fur, acdS, his $A$ and rps $U$ have revealed the complexity of their evolutionary relationships [21-26]. Based on assessment of conserved sequence indels, a new Paraburkholderia genus was created to include the diverse environmental isolates while Burkholderia sensu stricto includes B. mallei, B. pseudomallei, Bcc members and other pathogenic members [27]. The complicated taxonomy of Burkholderia sensu lato has attracted great attention, and large scale phylogenomic study has been suggested [28]. In accordance with this suggestion, a recent study systematically analyzed the conserved sequences in 92 Burkholderia sensu lato species and demonstrated the existence of 5 lineages: Burkholderia

(c) The Author(s). 2019 Open Access This article is distributed under the terms of the Creative Commons Attribution 4.0 International License (http://creativecommons.org/licenses/by/4.0/), which permits unrestricted use, distribution, and reproduction in any medium, provided you give appropriate credit to the original author(s) and the source, provide a link to the Creative Commons license, and indicate if changes were made. The Creative Commons Public Domain Dedication waiver (http://creativecommons.org/publicdomain/zero/1.0/) applies to the data made available in this article, unless otherwise stated. 
sensu stricto, Paraburkholderia, Caballeronia, the newly described genus Robbsia [29], and the lineage represented by Paraburkholderia rhizoxinica [30]. Assessment of the phylogenetic position of new isolates or samples containing Burkholderia or Paraburkholderia could be useful for further analysis, and there have been such reports based on molecular techniques such as PCR [22, 31-34], multi-locus sequence typing (MLST) [35-38] and qPCR assays [39-41]. As qPCR could sensitively quantitate the target and is accessible to more places compared with sequencing-based approaches, it has great application potentials in the phylogenetic studies of Burkholderia sensu lato.

Paraburkholderia caribensis (formerly Burkholderia caribensis) MBA4 is a bacterium with the ability to degrade environmental pollutant haloacids [42]. Besides the hydrolytic enzyme dehalogenase, membrane transporters that mediate active uptake of haloacids are also important for effective biodegradation [43-45]. Although structurally similar, haloacetate is transported with a different system compared with acetate [46]. We have recently revealed that $P$. caribensis MBA4 harbors two haloacids transporters, Deh4p and Dehp2, which show overlapping but not identical substrate specificities $[45,47]$. The expression of dehp2 is strictly regulated in response to the presence of haloacids in its growing environment, and the $\sim 100 \mathrm{bp}$ upstream non-coding region of dehp2 is highly conserved in Burkholderia sensu lato [48]. Three strains from other environmental Paraburkholderia species, namely P. caribensis LMG 18531, Paraburkholderia phymatum (formerly Burkholderia phymatum) STM815, and Paraburkholderia xenovorans (formerly Burkholderia xenovorans) LB400, gained the ability to degrade haloacids with the introduction of dehalogenase Deh4a, and haloacids-inducible haloacids transport activities were observed in accordance with haloacids-inducible expressions of dehp 2 orthologs, strongly suggesting their roles as haloacids transporters [16]. Dehp2 thus represents a group of conserved transporters in Burkholderia sensu lato, and the phylogenetic tree based on Dehp2 clearly show two clades corresponding well to Burkholderia sensu stricto and Paraburkholderia [43]. This provides the ground of exploiting Dehp2 for discrimination of Burkholderia sensu stricto and Paraburkholderia.

In this study, we tested the utilization of dehp2 as a phylogenetic marker for quick discrimination of putative pathogenic/opportunistic pathogenic Burkholderia sensu stricto and mainly environmental-derived Paraburkholderia. We designed qPCR assays that target the regions of dehp2 and 16S rRNA conserved in Burkholderia sensu stricto or Paraburkholderia and validated their performance in 12 strains of bacteria from Burkholderia sensu lato. The results showed that $\operatorname{deh} p 2$ could be used as a discriminative marker similarly as $16 \mathrm{~S}$ rRNA, and assays based on both markers produced more reliable results with high specificity and sensitivity. This is the first report on the utilization of a haloacids transporter as a discriminative marker in Burkholderia sensu lato, which will be useful for further clinical or biotechnological studies.

\section{Results \\ Quick detection of Burkholderia sensu stricto and Paraburkholderia by qPCR assays}

As described previously, both $16 \mathrm{~S}$ rRNA and dehp2 phylogenetic trees display two major groups which could discriminate Burkholderia sensu stricto and Paraburkholderia species [5, 43]. We first designed three pairs of qPCR primers based on 16S rRNA: 16S-F1/R1 to target the region conserved in Burkholderia sensu lato, 16S-F2/R2 to target the region conserved in Burkholderia sensu stricto, and 16S-F3/R3 to target the region conserved in Paraburkholderia. Similarly, we designed dehp2-F6/R6 and dehp2-F7/ R7 to target Burkholderia sensu stricto-specific and Paraburkholderia-specific regions of dehp2. To ensure that the primers cover all the sequence variations, we incorporated degenerate bases during primer design, which have been a common practice in microbial studies [49], such as microbial population taxonomy [50], diversity of functional genes related to antibiotic or arsenite resistance, etc. [51, 52].

We first analyzed the performances of the 5 pairs of primers against the 12 strains using a single strain as the template for each qPCR reaction (Fig. 1). For the Burkholderia sensu lato-conserved primer pair 16S-F1/R1, all 12 strains showed similar amplification efficiency. The Burkholderia sensu stricto-specific primer pair 16S-F2/R2 had statistically higher amplification efficiency with the 4 Burkholderia strains and C. glathei LMG 14190, while the Paraburkholderia-specific 16S-F3/R3 primer pair showed statistically higher amplification efficiency with the 7 Paraburkholderia strains. For dehp2, the Burkholderia sensu stricto-specific primer pair dehp2-F6/R6 had statistically higher amplification efficiency with the 4 Burkholderia strains, while the Paraburkholderia-specific dehp2-F7/R7 primer pair showed statistically higher amplification efficiency with the 7 Paraburkholderia strains and C. glathei LMG 14190. It should be pointed out that the amplification efficiency of qPCR assays will be affected by degenerate primers, but the amplification efficiencies of our discriminative qPCR assays show such dramatic differences between Burkholderia sensu stricto and Paraburkholderia group that they still enable effective discrimination of the two group even with the use of degenerate primers. The performances of primers targeting dehp2 were comparable to $16 \mathrm{~S}$ rRNA-based primers, suggesting dehp 2 could be used 


\section{S-F1/R1}

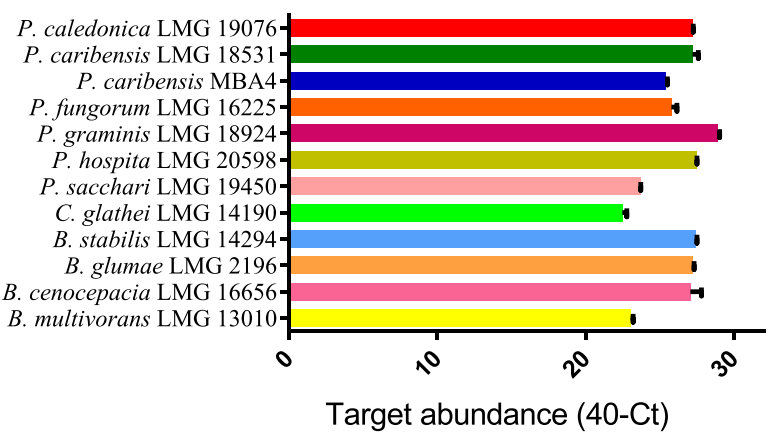

\section{S-F2/R2}

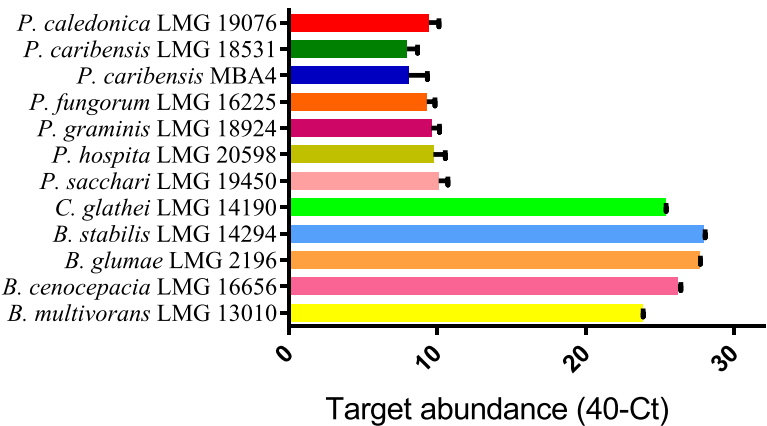

\section{dehp2-F6/R6}

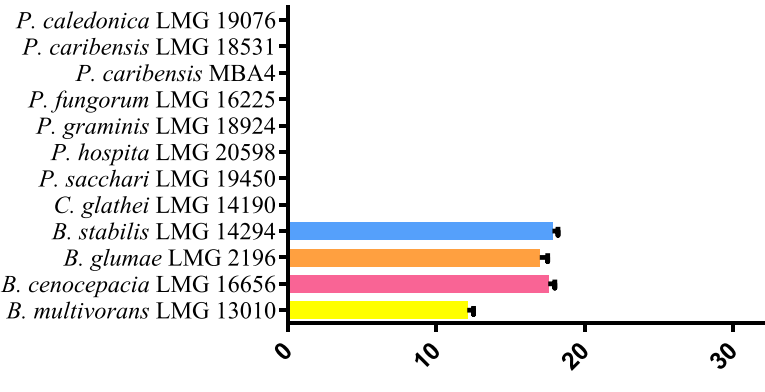

Target abundance (40-Ct)
16S-F3/R3

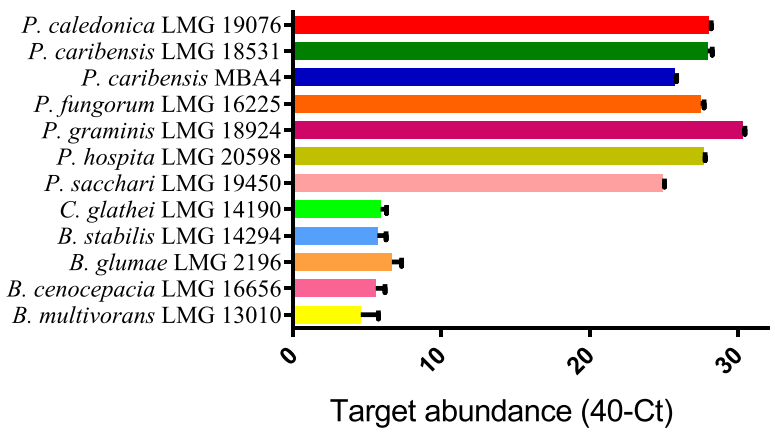

dehp2-F7/R7

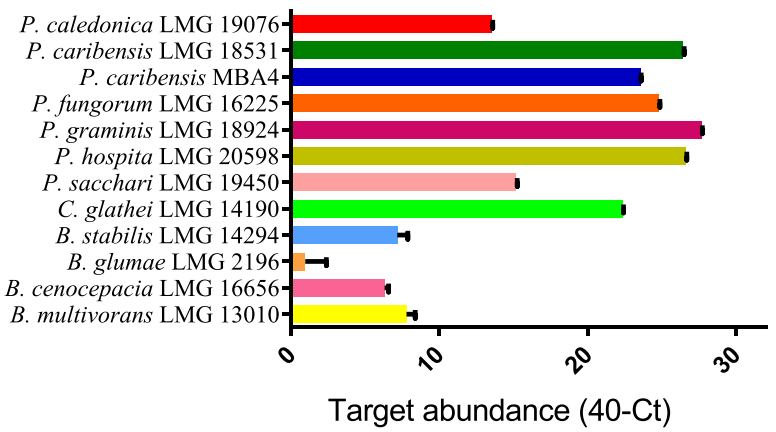

Fig. 1 qPCR assays to discriminate Burkholderia and Paraburkholderia species bacteria. qPCR analysis was carried out for the 12 strains with the 5 pairs of primers: 16S-F1/R1, 16S-F2/R2, 16S-F3/R3, dehp2-F6/R6 and dehp2-F7/R7. Target abundance was measured by 40 minus Ct value for each condition. Results shown are the means of three replicates with the error bars representing the standard deviations as a marker for discrimination of Burkholderia sensu stricto and Paraburkholderia.

The above results clearly showed that the 4 pairs of discriminative primers work well in regard to their ability to discriminate Burkholderia sensu stricto and Paraburkholderia. The interesting species is C. glathei, as qPCR patterns based on $16 \mathrm{~S}$ rRNA assays were similar to Burkholderia, while qPCR patterns based on dehp2 assays were similar to Paraburkholderia. This seemingly contradictory results showed the uniqueness of this species, which is supported by its recent transfer to a new genus Caballeronia [53].

\section{Specific discrimination of Burkholderia and Paraburkholderia from mixtures by qPCR assays}

The above results showed that the qPCR assays are able to discriminate whether the samples contain Burkholderia or Paraburkholderia species when we used a single bacterial species as the qPCR template. For environmental or clinical samples collected without further cultivation and 
isolation, it is common that they contain different bacterial species. To test whether our qPCR assays are capable of specific discrimination of Burkholderia or Paraburkholderia species, we mimicked such conditions by preparing mixtures of gDNA from known species. We first tested three conditions: equal concentration mixture of 2 Burkholderia species, mixture of 2 Paraburkholderia species, and mixture of 1 Burkholderia species and 1 Paraburkholderia species. Our qPCR assays could clearly tell whether there are only Burkholderia, only Paraburkholderia, or both genera in the samples, which are also consistent with values calculated from qPCR results of single species (Fig. 2a).

In addition to the equal concentration mixtures, we further assessed mixtures of Burkholderia and Paraburkholderia species at constant total concentration but different ratios. qPCR showed that for relative ratios of 1:125, 1:25, 1:5, 1:1, 5:1, 25:1 and 125:1 of B. cenocepacia LMG 16656 to $P$. caribensis LMG 18531, all the 4 pairs of discriminative primers exhibited specific detection (Fig. 2b). We then used the Delta-Ct values between the Burkholderia sensu stricto-specific and Paraburkholderia-specific primers (16S-F3/R3 vs. 16S-F2/R2, dehp2-F7/R7 vs. dehp2-F6/R6) to monitor their ability to discriminate the two genera. This approach could also avoid possible interference by other closely related bacteria. For the dynamic range tested, high correlation coefficients were observed for Delta-Ct values against different ratios of the two genera for both 16S rRNA and dehp2 (Fig. 2c). The results showed that our qPCR assays can specifically discriminate Burkholderia and Paraburkholderia species with a wide dynamic range of relative ratios.

Illustrative visualization of the qPCR data by hierarchical clustering $(\mathrm{HC})$ and principal component analysis (PCA) We then used HC and PCA to analyze the qPCR results for more straightforward visualization. For the qPCR data collected against single species or mixtures as described above, we first used $\mathrm{HC}$ to construct a heat-map. Both the

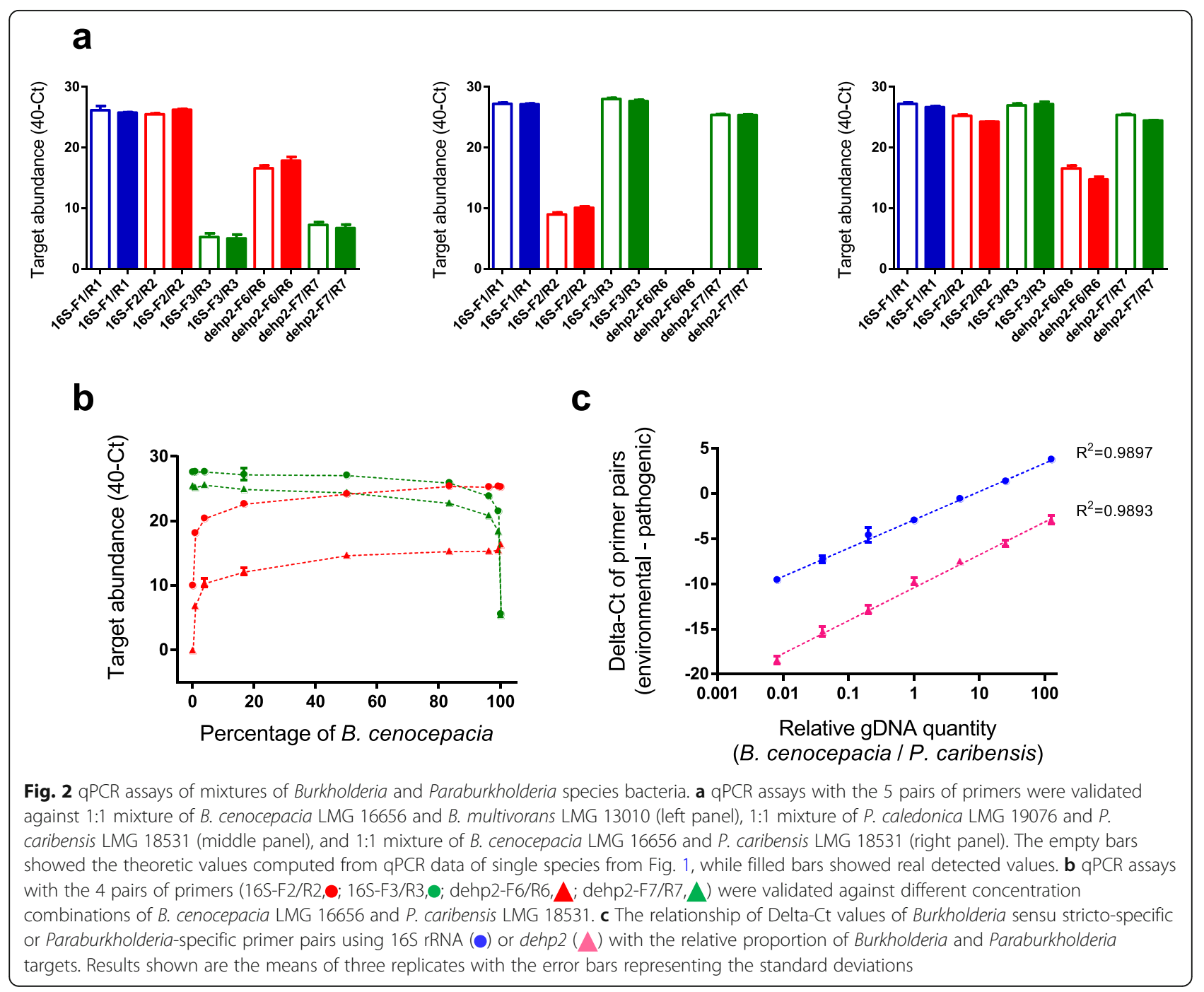


primer pairs and samples were clustered relevant to the genus they belong, facilitating easy interpretation of the results (Fig. 3a). The uniqueness of C. glathei LMG 14190 is also shown in the heat-map. For PCA score plot, the positions of the mixtures are straightforward display of their relative proportion of Burkholderia or Paraburkholderia (Fig. 3b). For PCA loading plot, the positions of the primer pairs also indicate whether they are targeting Burkholderia sensu lato-conserved, Burkholderia sensu stricto-specific or Paraburkholderia-specific region (Fig. 3c). In general, $\mathrm{HC}$ and PCA displays of the qPCR results facilitate easy detection of the presence and relative quantity of Burkholderia and Paraburkholderia species.

\section{High sensitivity of the qPCR assays for detection of Burkholderia and Paraburkholderia}

Our qPCR assays are able to specifically discriminate Burkholderia and Paraburkholderia species in complicated samples, and the amplification specificities of the 4 discriminative PCR assays were further confirmed by using gDNA of $E$. coli DH5 $\alpha$ as negative control (Additional file 1: Figure S1). We then checked their sensitivity at detecting the target species. We prepared serial diluted bacterial gDNA of Burkholderia and Paraburkholderia, and for a dynamic range of six orders of magnitude, all 4 pairs of primers worked consistently (Fig. 4). The targets were approaching several-copies to even single-copy per qPCR assay for the lowest concentration tested (calculated to be $<10$ copies $\mu^{-1}$ ), and all primer pairs were performing well except for Burkholderia sensu stricto-specific dehp2-F7/R7, which is relatively less efficient compared with the others. This is reasonable, as haloacids transporter gene is a good marker for environmental rather than pathogenic species. The above results showed that our qPCR assays are highly sensitive for detection of low-quantity of Burkholderia and Paraburkholderia target in the samples.

\section{Discussion}

Considering the pathogenic potentials of some species in Burkholderia sensu lato, it is useful to first have a quick assessment before further analysis such as sequencing, especially when there is a large number of specimens. As some bacteria may not be cultured successfully, qPCR assay should be more sensitive than culture-dependent detection methods. On the other hand, although high throughput sequencing is more powerful to give a global view of genomes, qPCR can actually show very consistent results towards specific targets at much lower cost [54]. Moreover, qPCR is also easier to handle and accessible to more places compared with sequencing-based identification approaches. With these advantages, there have been many qPCR based methods for detection or discrimination purposes in Burkholderia sensu lato. For example, qPCR assay based on Type III Secretion System enabled quick and accurate identification of $B$. pseudomallei [39], and qPCR assay Bu550 that targets a $7 \mathrm{~kb}$ locus was able to discriminate $B$. ubonensis from its close neighbor B. pseudomallei [55]. Multi-target qPCR assays were able to detect the presence of Bcc members at the resolution of species from sputum specimens [40]. Our study represents the first application of a haloacids transporter gene to discriminate Burkholderia and Paraburkholderia, which will be useful for not only detecting pathogenic species but also screening environmental species that can be exploited for bioremediation of haloacids.

It has been suggested that phylogenetic relationship could not be reliably established based on single gene [56], and the inclusion of more independent targets increased the sensitivity compared with single target method for identification of $B$. pseudomallei $[41,57]$. In this study, we also considered this issue and utilized both the haloacids transporter gene dehp 2 and $16 \mathrm{~S}$ rRNA. Indeed, there were in-consistence between results obtained from assays based on dehp 2 and $16 \mathrm{~S}$ rRNA in regard to $C$. glathei, which was transferred to a novel genus recently [53], further supporting the reliability of the assays. Moreover, our qPCR assays were designed to target regions conserved in both pathogenic Burkholderia sensu stricto species and environmental Paraburkholderia species, and the Delta-Ct values between Burkholderia sensu stricto-specific and Paraburkholderia-specific assays could be utilized to calculate the relative ratio of the two genera in mixtures. The combination of qPCR assays that target both Burkholderia-specific and Paraburkholderia-specific regions in dehp2 and 16S rRNA could rule out possible inference by other related environmental bacteria. High sensitivity and specificity of such assays were observed for both $\operatorname{dehp} 2$ and 16S rRNA over a broad dynamic range of mixtures from both genera.

Our previous work has established Dehp2 as a haloacids transporter [43, 47], and the successful application of this gene to discriminate Burkholderia sensu stricto and Paraburkholderia further proved its importance for Burkholderia sensu lato. As haloacids are not the natural nutrients for pathogenic Burkholderia species and even some of the environmental Paraburkholderia species, the presence of this transporter showed gene expression rewiring and adaption potentials of bacteria to their living environment. Further analysis of this transporter gene among the two genera, such as evolutionary analysis of key amino acid residues and comparative assessment of the promoter regions between pathogenic and environmental species should provide precious clues for understanding of the transport mechanisms and technological exploitation of relevant species to efficiently degrade haloacids without causing pathogenic risks. 


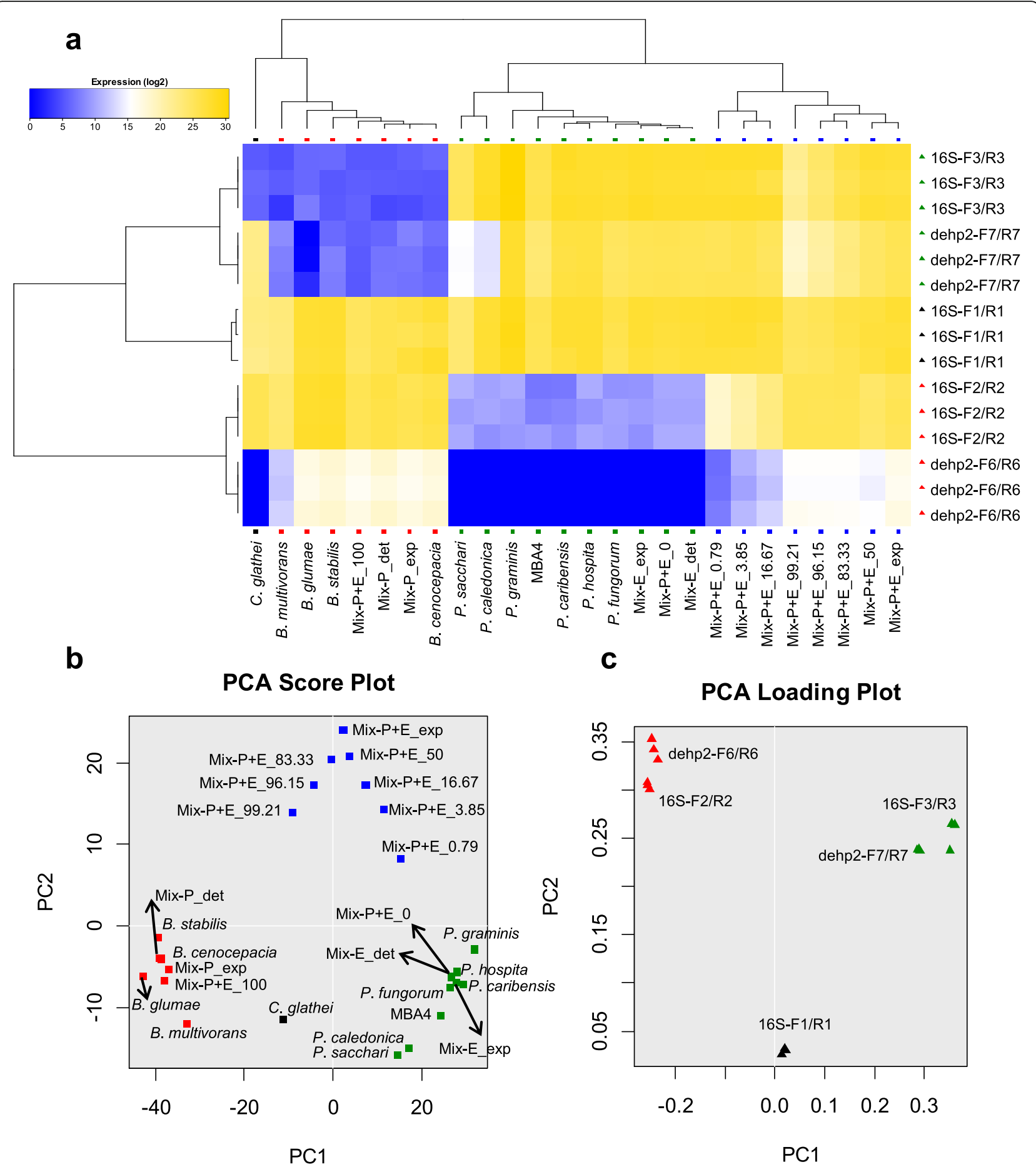

Fig. 3 Hierarchical clustering $(\mathrm{HC})$ and principal component analysis (PCA) of the qPCR assays. The data used in the Figs. 1 and 2 were combined and further analyzed with HC and PCA. a Heat-map based on HC. The samples and genes were differently colored based on the genus they belong. PCA score plot (b) and PCA loading plot (c) of the results are shown. For sample groups: Burkholderia, ; Paraburkholderia, , Burkholderia +

Paraburkholderia, other, For gene groups: Burkholderia sensu stricto-specific, $\mathbf{\Lambda}$; Paraburkholderia-specific, $\mathbf{\Lambda}$; Burkholderia sensu lato-conserved, For sample names, 'Mix-P', 'Mix-E' and 'Mix-E + $P^{\prime}$ indicate mixture of two Burkholderia species, two Paraburkholderia species, and one Burkholderia and one Paraburkholderia species, respectively; '_exp' and '_det' indicate expected and detected values; the numbers after 'Mix-E + $P$ ' indicate the percentage of $B$. cenocepacia LMG 16656 in the mixtures. All three replicates of qPCR are shown 


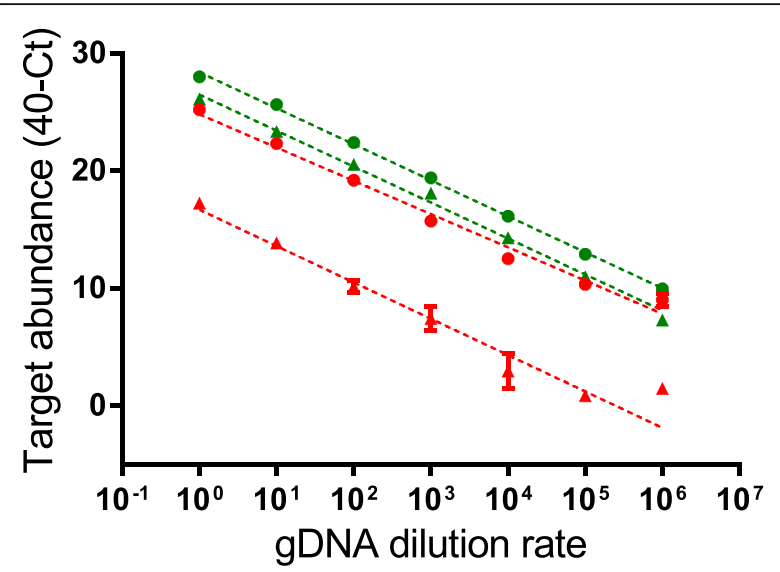

Fig. 4 Detection sensitivity of the GPCR assays. The sensitivities of the 4 pairs of primers (16S-F2/R2, ; 16S-F3/R3, $\bullet$; dehp2-F6/R6, dehp2-F7/R7, $)$ were checked against serial diluted bacterial gDNA $\left(\sim 50 \mathrm{ng} \mathrm{H}^{-1}\right.$ for both $B$. cenocepacia LMG 16656 and $P$. caribensis LMG 18531) at the following rate: $10^{1}, 10^{2}, 10^{3}, 10^{4}, 10^{5}$ and $10^{6}$. Results shown are the means of three replicates with the error bars representing the standard deviations

It should be pointed out that, however, qPCR assay is just a first step for full understanding of the bacteria or samples. As has been cautioned, phylogenetic positions not necessarily confirm whether a Burkholderia or Paraburkholderia species is pathogenic or not $[5,6,58]$. The results based on this qPCR assay should raise our attention to putative pathogenic species that must be handled carefully, and the samples suggested to be in the environmental group should also be systematically assessed before wide technological applications. Another fact that should be emphasized is that we only tested the qPCR assays in 12 bacterial strains, which represent a relatively small sampling of the $>100$ of strains from Burkholderia sensu lato. Further assessment of the assays in a larger sample pool of Burkholderia sensu lato members will be beneficial for the research field and broad applications of the assays.

\section{Conclusions}

In this study we designed qPCR assays based on haloacids transporter Dehp2 as well as $16 \mathrm{~S}$ rRNA, which enable quick discrimination of Burkholderia species and Paraburkholderia species with high sensitivity and specificity. Results obtained with the qPCR assays will facilitate more specific handling in regard to the putative pathogenicity of the samples and also exploitation of relevant species for haloacids bioremediation.

\section{Methods}

Bacterial strains and extraction of genomic DNA

We used 12 strains from 11 species from Burkholderia sensu lato in this study, namely Paraburkholderia caledonica LMG 19076, P. caribensis LMG 18531, P. caribensis MBA4, Paraburkholderia fungorum LMG 16225, Paraburkholderia graminis LMG 18924, Paraburkholderia hospita LMG 20598, Paraburkholderia sacchari LMG 19450, Caballeronia glathei LMG 14190, Burkholderia stabilis LMG 14294, Burkholderia glumae LMG 2196, B. cenocepacia LMG 16656 and B. multivorans LMG 13010, which were gifts from Molecular Microbiology Laboratory of The University of Hong Kong (Table 1). Genomic DNAs (gDNAs) from the 12 strains were extracted with a G-spin ${ }^{\mathrm{tm}}$ Genomic DNA Extraction Kit (iNtRON). The concentrations of the

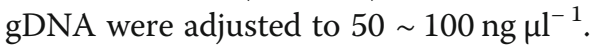

\section{Primer design of $16 \mathrm{~S}$ rRNA and dehp2}

The 16S rRNA and dehp 2 sequences from the above described strains were retrieved from NCBI. For the haloacids transporter gene dehp2, the sequences are available in 6 species (P. caribensis, P. fungorum, B. stabilis, B. glumae, $B$. cenocepacia, and $B$. multivorans), and the sequences are currently unknown in the other 5 species ( $P$. caledonica, $P$. graminis, $P$. hospita, $P$. sacchari and $C$. glathei). The sequences of $16 \mathrm{~S}$ rRNA or dehp 2 were aligned using

Table 1 Bacterial strains used in this study

\begin{tabular}{|c|c|c|}
\hline Bacterial strains & Description & References \\
\hline E. coli DH5a & $\begin{array}{l}\text { Negative control for } \\
\text { primer validation }\end{array}$ & Takara \\
\hline $\begin{array}{l}\text { P. caledonica } \\
\text { LMG } 19076\end{array}$ & $\begin{array}{l}\text { Bacterium isolated from } \\
\text { the rhizosphere }\end{array}$ & [61] \\
\hline $\begin{array}{l}\text { P. caribensis } \\
\text { LMG } 18531\end{array}$ & $\begin{array}{l}\text { Exopolysaccharide-producing } \\
\text { bacterium isolated } \\
\text { from vertisol }\end{array}$ & {$[62,63]$} \\
\hline $\begin{array}{l}\text { P. caribensis } \\
\text { MBA4 }\end{array}$ & $\begin{array}{l}\text { Haloacids-degrading bacterium } \\
\text { isolated from soil }\end{array}$ & {$[42,64,65]$} \\
\hline $\begin{array}{l}\text { P. fungorum } \\
\text { LMG } 16225\end{array}$ & $\begin{array}{l}\text { Bacterium isolated from } \\
\text { the white-rot fungus }\end{array}$ & [61] \\
\hline $\begin{array}{l}\text { P. graminis } \\
\text { LMG } 18924\end{array}$ & $\begin{array}{l}\text { Bacterium isolated from rhizosphere } \\
\text { of grasses }\end{array}$ & [66] \\
\hline $\begin{array}{l}\text { P. hospita LMG } \\
20598\end{array}$ & $\begin{array}{l}\text { Bacterium isolated from B-horizon } \\
\text { soil }\end{array}$ & [67] \\
\hline $\begin{array}{l}\text { P. sacchari } \\
\text { LMG } 19450\end{array}$ & $\begin{array}{l}\text { Polyhydroxyalkanoate-accumulating } \\
\text { bacterium isolated from soil }\end{array}$ & [68] \\
\hline $\begin{array}{l}\text { C. glathei } \\
\text { LMG } 14190\end{array}$ & Bacterium isolated from lateritic soil & {$[53,66,69]$} \\
\hline $\begin{array}{l}\text { B. stabilis } \\
\text { LMG } 14294\end{array}$ & $\begin{array}{l}\text { Pathogenic bacterium isolated from } \\
\text { sputum of a cystic fibrosis patient, } \\
\text { Bcc member }\end{array}$ & [70] \\
\hline $\begin{array}{l}\text { B. glumae } \\
\text { LMG } 2196\end{array}$ & Plant pathogen & [71] \\
\hline $\begin{array}{l}\text { B. cenocepacia } \\
\text { LMG } 16656\end{array}$ & $\begin{array}{l}\text { Pathogenic bacterium isolated from } \\
\text { a cystic fibrosis patient, Bcc member }\end{array}$ & [72] \\
\hline $\begin{array}{l}\text { B. multivorans } \\
\text { LMG } 13010\end{array}$ & $\begin{array}{l}\text { Pathogenic bacterium isolated from } \\
\text { sputum of a cystic fibrosis patient, } \\
\text { Bcc member }\end{array}$ & [73] \\
\hline
\end{tabular}


ClustalW [59]. Three pairs of qPCR primers were then designed based on 16S rRNA: 16S-F1/R1 to target the region conserved in Burkholderia sensu lato, 16S-F2/R2 to target the region conserved in Burkholderia sensu stricto, and 16S-F3/R3 to target the region conserved in Paraburkholderia. We designed dehp2-F6/R6 and dehp2-F7/R7 to target Burkholderia sensu stricto-specific and Paraburkholderia-specific regions of dehp2. The sequences of the 5 pairs of primers are shown in Table 2.

\section{qPCR}

qPCR was carried out using $\mathrm{SYBR}^{\circ}$ Premix Ex Taq ${ }^{\mathrm{Tm}}$ (Clontech) on the StepOnePlus system (Applied Biosystems) with a two-step method: initial denaturation of $95^{\circ} \mathrm{C}$ for $30 \mathrm{~s} ; 40$ cycles of $95^{\circ} \mathrm{C}$ for $5 \mathrm{~s}$ and $66^{\circ} \mathrm{C}$ for $30 \mathrm{~s}$. A melting curve program was also included to verify the specificity of the amplified products. Three replicates were set up for each condition, and negative controls were also included to monitor possible contaminations. To measure the amplification efficiency, 40 minus $\mathrm{Ct}$ values were used, which represent logarithmic transformed target abundance as previous described $[54,60]$. To check the ability of combination of primer pairs to discriminate Burkholderia sensu stricto and Paraburkholderia, the difference between $\mathrm{Ct}$ values derived from $16 \mathrm{~S}$ rRNA primers $\left(\mathrm{Ct}_{16 \mathrm{~S}-\mathrm{F} 3 / \mathrm{R} 3}\right.$ - $\left.\mathrm{Ct}_{16 \mathrm{~S}-\mathrm{F} 2 / \mathrm{R} 2}\right)$, or between those derived from dehp2 primers $\left(\mathrm{Ct}_{\mathrm{dehp2-F7/R7}}-\mathrm{Ct}_{\mathrm{dehp2-F6/R6}}\right)$ were calculated. For qPCR reactions, besides the single strains analyzed, we also tested mixtures of different strains. To check the sensitivity of qPCR assays, serial diluted gDNA of B. cenocepacia LMG 16656 was used as template for 16S-F2/R2 and dehp2-F6/R6, while serial diluted gDNA of $P$. caribensis LMG 18531 was used as template for 16S-F3/R3 and dehp2-F7/R7.

Table 2 Primers used in this study

\begin{tabular}{ll}
\hline Primers & Sequence $\left(5^{\prime} \text { to } 3^{\prime}\right)^{\text {a }}$ \\
\hline 16S-F1 & GGTAATACGTAGGGTGRAGCGTT \\
16S-R1 & CACMAATGCAGTTCCCAGGTTRAG \\
16S-F2 & GGAGGAATACCGATGGCGAAGG \\
16S-R2 & TTACTAAGGAAATGAATCCCCAACAAC \\
16S-F3 & ACAAGCGGTGGATGATGTGGAT \\
16S-R3 & TGTGTAYGGCTCCCTTCGG \\
dehp2-F6 & RCAYTCGCCGATGACGRS \\
dehp2-R6 & GGARAAGAAGCTCTTGCTGATRT \\
dehp2-F7 & RCMTGGGGCTGGCGCATT \\
dehp2-R7 & GTCCGGRTSGCGATCACGAC \\
\hline
\end{tabular}

${ }^{\mathrm{a}}$ Degenerated bases are shown in bold

\section{Visualization of data by HC and PCA}

$\mathrm{HC}$ and PCA analysis of the qPCR data were performed using the SINGuLAR ${ }^{\text {tu }}$ Analysis Toolset $\mathrm{R}$ package (Fluidigm).

\section{Additional file}

Additional file 1: Figure S1 Validation of the amplification specificity of the discriminative PCR primers. (PDF $226 \mathrm{~kb}$ )

\section{Abbreviations}

Bcc: Burkholderia cepacia complex; HC: Hierarchical clustering; PCA: Principal component analysis

\section{Acknowledgements}

We would like to thank Dr. Jimmy S.H. Tsang and Mr. Ka-Fai Kong from The University of Hong Kong for critical discussions and providing the genomic DNAs.

\section{Funding}

This work is supported in part by the National Natural Science Foundation of China (81802806, 81472621 and 81402329), National Program on Key Research Project of China (2016YFC0902701, Precision Medicine), Medical and Engineering Crossover Fund of SJTU (YG2016QN71, YG2017MS67), the University of Sydney \& Shanghai Jiao Tong University Joint Research Alliance (SJTU-USYD Translational Medicine Fund-Systems Biomedicine AF6260003), and funding from Key Laboratory of Systems Biomedicine (Ministry of Education) (KLSB2017QN-03). The funding bodies didn't involve in the design of the study, collection, analysis, interpretation of data, or writing the manuscript.

\section{Availability of data and materials}

The authors declare that data supporting the findings are included in the article, and materials are available upon request.

\section{Authors' contributions}

$\mathrm{XS}$ and $\mathrm{ZH}$ conceived and designed the study. XS, ZL and JW carried out experiments. XS, YS, RL, XZ analyzed data. XS interpreted the results and drafted the manuscript. All authors read and approved final version of the manuscript.

Ethics approval and consent to participate

Not applicable.

Consent for publication

Not applicable.

Competing interests

The authors declare that they have no competing interests.

\section{Publisher's Note}

Springer Nature remains neutral with regard to jurisdictional claims in published maps and institutional affiliations.

\section{Author details \\ ${ }^{1}$ Key Laboratory of Systems Biomedicine (Ministry of Education), Shanghai Center for Systems Biomedicine, Shanghai Jiao Tong University, Shanghai, China. ${ }^{2}$ Shanghai Quality Safety Centre of Agricultural Products, Shanghai, China.}

Received: 30 November 2017 Accepted: 31 January 2019

Published online: 11 February 2019

\section{References}

1. Coenye T, Vandamme P. Diversity and significance of Burkholderia species occupying diverse ecological niches. Environ Microbiol. 2003;5(9):719-29. 
2. Compant S, Nowak J, Coenye T, Clement C, Ait Barka E. Diversity and occurrence of Burkholderia spp. in the natural environment. FEMS Microbiol Rev. 2008;32(4):607-26.

3. Vial L, Chapalain A, Groleau MC, Deziel E. The various lifestyles of the Burkholderia cepacia complex species: a tribute to adaptation. Environ Microbiol. 2011;13(1):1-12.

4. da Silva K, Cassetari Ade S, Lima AS, De Brandt E, Pinnock E, Vandamme P, Moreira FM. Diazotrophic Burkholderia species isolated from the Amazon region exhibit phenotypical, functional and genetic diversity. Syst Appl Microbiol. 2012;35(4):253-62.

5. Depoorter E, Bull MJ, Peeters C, Coenye T, Vandamme P, Mahenthiralingam E. Burkholderia: an update on taxonomy and biotechnological potential as antibiotic producers. Appl Microbiol Biotechnol. 2016;100(12):5215-29.

6. Eberl L, Vandamme P. Members of the genus Burkholderia: good and bad guys. F1000Res. 2016;5:1007.

7. Ussery DW, Kiil K, Lagesen K, Sicheritz-Ponten T, Bohlin J, Wassenaar TM. The genus Burkholderia: analysis of 56 genomic sequences. Genome Dyn. 2009;6: 140-57

8. Galyov EE, Brett PJ, DeShazer D. Molecular insights into Burkholderia pseudomallei and Burkholderia mallei pathogenesis. Annu Rev Microbiol. 2010;64:495-517.

9. Chewapreecha C, Holden MT, Vehkala M, Valimaki N, Yang Z, Harris SR, Mather AE, Tuanyok A, De Smet B. Le hello S et al: global and regional dissemination and evolution of Burkholderia pseudomallei. Nat Microbiol. 2017;2:16263.

10. Limmathurotsakul D, Golding N, Dance DA, Messina JP, Pigott DM, Moyes CL, Rolim DB, Bertherat E, Day NP, Peacock SJ, et al. Predicted global distribution of Burkholderia pseudomallei and burden of melioidosis. Nat Microbiol. 2016;1:15008.

11. Tsang JS. Molecular biology of the Burkholderia cepacia complex. Adv Appl Microbiol. 2004;54:71-91.

12. Chiarini L, Bevivino A, Dalmastri C, Tabacchioni S, Visca P. Burkholderia cepacia complex species: health hazards and biotechnological potential. Trends Microbiol. 2006;14(6):277-86.

13. Mahenthiralingam E, Baldwin A, Dowson CG. Burkholderia cepacia complex bacteria: opportunistic pathogens with important natural biology. J Appl Microbiol. 2008;104(6):1539-51.

14. Vandamme P, Dawyndt P. Classification and identification of the Burkholderia cepacia complex: past, present and future. Syst Appl Microbiol. 2011;34(2):87-95.

15. O'Sullivan LA, Mahenthiralingam E. Biotechnological potential within the genus Burkholderia. Lett Appl Microbiol. 2005;41(1):8-11.

16. Su X, Deng L, Kong KF, Tsang JS. Enhanced degradation of haloacid by heterologous expression in related Burkholderia species. Biotechnol Bioeng. 2013;110(10):2687-96.

17. Paungfoo-Lonhienne $C$, Lonhienne TG, Yeoh YK, Donose BC, Webb RI, Parsons J, Liao W, Sagulenko E, Lakshmanan P, Hugenholtz P, et al. Crosstalk between sugarcane and a plant-growth promoting Burkholderia species. Sci Rep. 2016;6:37389.

18. Estrada-de los Santos P, Rojas-Rojas FU, Tapia-García EY, Vásquez-Murrieta MS, Hirsch AM. To split or not to split: an opinion on dividing the genus Burkholderia. Ann Microbiol. 2016;66(3):1303-14.

19. Suarez-Moreno ZR, Caballero-Mellado J, Coutinho BG, Mendonca-Previato L, James EK, Venturi V. Common features of environmental and potentially beneficial plant-associated Burkholderia. Microb Ecol. 2012;63(2):249-66.

20. Gyaneshwar P, Hirsch AM, Moulin L, Chen WM, Elliott GN, Bontemps C. Estrada-de Los Santos P, gross E, dos reis FB, Sprent II et al: legumenodulating betaproteobacteria: diversity, host range, and future prospects. Mol Plant-Microbe Interact. 2011;24(11):1276-88.

21. Mahenthiralingam E, Bischof J, Byrne SK, Radomski C, Davies JE, Av-Gay Y, Vandamme P. DNA-based diagnostic approaches for identification of Burkholderia cepacia complex, Burkholderia vietnamiensis, Burkholderia multivorans, Burkholderia stabilis, and Burkholderia cepacia genomovars I and III. J Clin Microbiol. 2000;38(9):3165-73.

22. Payne GW, Vandamme P, Morgan SH, LiPuma JJ, Coenye T, Weightman AJ, Jones $\mathrm{TH}$, Mahenthiralingam E. Development of a recA gene-based identification approach for the entire Burkholderia genus. Appl Environ Microbiol. 2005:71(7):3917-27.

23. Lynch KH, Dennis JJ. Development of a species-specific fur gene-based method for identification of the Burkholderia cepacia complex. J Clin Microbiol. 2008;46(2):447-55.
24. Onofre-Lemus J, Hernandez-Lucas I, Girard L, Caballero-Mellado J. ACC (1-aminocyclopropane-1-carboxylate) deaminase activity, a widespread trait in Burkholderia species, and its growth-promoting effect on tomato plants. Appl Environ Microbiol. 2009:75(20):6581-90.

25. Papaleo MC, Perrin E, Maida I, Fondi M, Fani R, Vandamme P. Identification of species of the Burkholderia cepacia complex by sequence analysis of the hisA gene. J Med Microbiol. 2010;59(Pt 10):1163-70.

26. Frickmann $H$, Neubauer $H$, Loderstaedt $U$, Derschum $H$, Hagen RM. rpsU-based discrimination within the genus Burkholderia. Eur J Microbiol Immunol. 2014;4(2):106-16.

27. Sawana A, Adeolu M, Gupta RS. Molecular signatures and phylogenomic analysis of the genus Burkholderia: proposal for division of this genus into the emended genus Burkholderia containing pathogenic organisms and a new genus Paraburkholderia gen. nov. harboring environmental species. Front Genet. 2014:5:429.

28. de Lajudie PM, Young JPW. International committee on systematics of prokaryotes Subcommittee for the Taxonomy of Rhizobium and Agrobacterium minutes of the meeting, Budapest, 25 august 2016. Int J Syst Evol Microbiol. 2017:67(7):2485-94.

29. Lopes-Santos L, Castro DBA, Ferreira-Tonin M, Correa DBA, Weir BS, Park D, Ottoboni LMM, Neto JR, Destefano SAL. Reassessment of the taxonomic position of Burkholderia andropogonis and description of Robbsia andropogonis gen. Nov., comb. nov. Antonie Van Leeuwenhoek. 2017;110(6):727-36.

30. Beukes CW, Palmer M, Manyaka P, Chan WY, Avontuur JR, van Zyl E, Huntemann M, Clum A, Pillay M, Palaniappan K, et al. Genome data provides high support for generic boundaries in Burkholderia Sensu Lato. Front Microbiol. 2017:8:1154

31. Wong-Villarreal A, Caballero-Mellado J. Rapid identification of nitrogen-fixing and legume-nodulating Burkholderia species based on PCR 165 rRNA species-specific oligonucleotides. Syst Appl Microbiol. 2010;33(1):35-43.

32. Peeters $C$, Daenekindt S, Vandamme P. PCR detection of Burkholderia multivorans in water and soil samples. BMC Microbiol. 2016;16(1):184.

33. Koh SF, Tay ST, Sermswan R, Wongratanacheewin S, Chua KH, Puthucheary SD. Development of a multiplex PCR assay for rapid identification of Burkholderia pseudomallei, Burkholderia thailandensis, Burkholderia mallei and Burkholderia cepacia complex. J Microbiol Meth. 2012;90(3):305-8.

34. Zakharova I, Teteryatnikova N, Toporkov A, Viktorov D. Development of a multiplex PCR assay for the detection and differentiation of Burkholderia pseudomallei, Burkholderia mallei, Burkholderia thailandensis, and Burkholderia cepacia complex. Acta Trop. 2017;174:1-8.

35. Baldwin A, Mahenthiralingam E, Thickett KM, Honeybourne D, Maiden MC Govan JR, Speert DP, Lipuma JJ, Vandamme P, Dowson CG. Multilocus sequence typing scheme that provides both species and strain differentiation for the Burkholderia cepacia complex. J Clin Microbiol. 2005; 43(9):4665-73.

36. Spilker T, Baldwin A, Bumford A, Dowson CG, Mahenthiralingam E, LiPuma JJ. Expanded multilocus sequence typing for Burkholderia species. J Clin Microbiol. 2009:47(8):2607-10.

37. Estrada-de los Santos P, Vinuesa P, Martinez-Aguilar L, Hirsch AM, CaballeroMellado J. Phylogenetic analysis of Burkholderia species by multilocus sequence analysis. Curr Microbiol. 2013;67(1):51-60.

38. Price EP, MacHunter B, Spratt BG, Wagner DM, Currie BJ, Sarovich DS. Improved multilocus sequence typing of Burkholderia pseudomallei and closely related species. J Med Microbiol. 2016;65(9):992-7.

39. Novak RT, Glass MB, Gee JE, Gal D, Mayo MJ, Currie BJ, Wilkins PP. Development and evaluation of a real-time PCR assay targeting the type III secretion system of Burkholderia pseudomallei. J Clin Microbiol. 2006;44(1):85-90.

40. Martinucci M, Roscetto E, lula VD, Votsi A, Catania MR, De Gregorio E. Accurate identification of members of the Burkholderia cepacia complex in cystic fibrosis sputum. Lett Appl Microbiol. 2016;62(3):221-9.

41. Gohler A, Trung TT, Hopf V, Kohler C, Hartleib J, Wuthiekanun V, Peacock SJ, Limmathurotsakul D, Tuanyok A, Steinmetz I. Multitarget quantitative PCR improves detection and predicts cultivability of the pathogen Burkholderia pseudomallei. Appl Environ Microbiol. 2017;83(8):e03212-6.

42. Tsang JSH, Sallis PJ, Bull AT, Hardman DJ. A monobromoacetate dehalogenase from Pseudomonas cepacia MBA4. Arch Microbiol. 1988; 150(5):441-6.

43. Su X, Li R, Kong KF, Tsang JS. Transport of haloacids across biological membranes. Biochim Biophys Acta. 2016;1858(12):3061-70. 
44. Jing NH, Wahab RA, Hamdan S, Huyop F. Cloning and DNA sequence analysis of the haloalkanoic permease uptake gene from Rhizobium sp. RC1. Biotechnology. 2010;9(3):319-25.

45. Yu M, Faan YW, Chung WY, Tsang JS. Isolation and characterization of a novel haloacid permease from Burkholderia cepacia MBA4. Appl Environ Microbiol. 2007;73(15):4874-80.

46. Su X, Kong KF, Tsang JS. Transports of acetate and haloacetate in Burkholderia species MBA4 are operated by distinct systems. BMC Microbiol. 2012;12:267.

47. Su X, Tsang JS. Existence of a robust haloacid transport system in a Burkholderia species bacterium. Biochim Biophys Acta. 2013;1828(2):187-92.

48. Su X, Li R, Tsang JS. The 228 bp upstream non-coding region of haloacids transporter gene dehp2 has regulated promoter activity. Gene. 2016;593(2):322-9.

49. Telenius H, Carter NP, Bebb CE, Nordenskjold M, Ponder BA, Tunnacliffe A. Degenerate oligonucleotide-primed PCR: general amplification of target DNA by a single degenerate primer. Genomics. 1992;13(3):718-25.

50. Hugerth LW, Wefer HA, Lundin S, Jakobsson HE, Lindberg M, Rodin S, Engstrand $L$, Andersson AF. DegePrime, a program for degenerate primer design for broad-taxonomic-range PCR in microbial ecology studies. Appl Environ Microbiol. 2014;80(16):5116-23.

51. Cai L, Liu G, Rensing C, Wang G. Genes involved in arsenic transformation and resistance associated with different levels of arsenic-contaminated soils. BMC Microbiol. 2009;9:4.

52. Fouhy F, Ross RP, Fitzgerald GF, Stanton C, Cotter PD. A degenerate PCRbased strategy as a means of identifying homologues of aminoglycoside and beta-lactam resistance genes in the gut microbiota. BMC Microbiol. 2014;14:25.

53. Dobritsa AP, Samadpour M. Transfer of eleven species of the genus Burkholderia to the genus Paraburkholderia and proposal of Caballeronia gen. Nov. to accommodate twelve species of the genera Burkholderia and Paraburkholderia. Int J Syst Evol Microbiol. 2016;66(8):2836-46.

54. Su X, Shi Y, Zou X, Lu Z-N, Xie G, Yang JYH, Wu C-C, Cui X-F, He K-Y, Luo Q, et al. Single-cell RNA-Seq analysis reveals dynamic trajectories during mouse liver development. BMC Genomics. 2017;18:946.

55. Price EP, Sarovich DS, Webb JR, Ginther JL, Mayo M, Cook JM, Seymour ML, Kaestli M, Theobald V, Hall CM, et al. Accurate and rapid identification of the Burkholderia pseudomallei near-neighbour, Burkholderia ubonensis, using realtime PCR. PLoS One. 2013;8(8):e71647.

56. Tayeb LA, Lefevre M, Passet V, Diancourt L, Brisse S, Grimont PA Comparative phylogenies of Burkholderia, Ralstonia, Comamonas, Brevundimonas and related organisms derived from $r p o B$, gyrB and rrs gene sequences. Res Microbiol. 2008;159(3):169-77.

57. Trung TT, Hetzer A, Gohler A, Topfstedt E, Wuthiekanun V, Limmathurotsakul D, Peacock SJ, Steinmetz I. Highly sensitive direct detection and quantification of Burkholderia pseudomallei bacteria in environmental soil samples by using realtime PCR. Appl Environ Microbiol. 2011;77(18):6486-94.

58. Peeters C, Meier-Kolthoff JP, Verheyde B, De Brandt E, Cooper VS, Vandamme P. Phylogenomic study of Burkholderia glathei-like organisms, proposal of 13 novel Burkholderia species and emended descriptions of Burkholderia sordidicola, Burkholderia zhejiangensis, and Burkholderia grimmiae. Front Microbiol. 2016;7:877.

59. Larkin MA, Blackshields G, Brown NP, Chenna R, McGettigan PA, McWilliam H, Valentin F, Wallace IM, Wilm A, Lopez R, et al. Clustal W and Clustal X version 2.0. Bioinformatics. 2007:23(21):2947-8.

60. Livak KJ, Wills QF, Tipping AJ, Datta K, Mittal R, Goldson AJ, Sexton DW, Holmes CC. Methods for aPCR gene expression profiling applied to 1440 lymphoblastoid single cells. Methods. 2013;59(1):71-9.

61. Coenye T, Laevens S, Willems A, Ohlen M, Hannant W, Govan JR, Gillis M, Falsen E, Vandamme P. Burkholderia fungorum sp. nov. and Burkholderia caledonica sp. nov., two new species isolated from the environment, animals and human clinical samples. Int J Syst Evol Microbiol. 2001;51(Pt 3):1099-107.

62. Achouak W, Christen R, Barakat M, Martel MH, Heulin T. Burkholderia caribensis sp. nov., an exopolysaccharide-producing bacterium isolated from vertisol microaggregates in Martinique. Int J Syst Bacteriol. 1999:49:787-94

63. Pan Y, Kong KF, Tsang JS. Complete genome sequence of the exopolysaccharide-producing Burkholderia caribensis type strain MWAP64. Genome Announc. 2016:4(1):e01636-15.

64. Pan Y, Kong KF, Tsang JS. Draft genome sequence of the haloaciddegrading Burkholderia caribensis strain MBA4. Genome Announc. 2014;2(1): e00047-14
65. Pan Y, Kong KF, Tsang JS. Complete genome sequence and characterization of the haloacid-degrading Burkholderia caribensis MBA4. Stand Genomic Sci. 2015;10:114

66. Viallard V, Poirier I, Cournoyer B, Haurat J, Wiebkin S, Ophel-Keller K, Balandreau J. Burkholderia graminis sp. nov., a rhizospheric Burkholderia species, and reassessment of [Pseudomonas] phenazinium, [Pseudomonas] pyrrocinia and [Pseudomonas] glathei as Burkholderia. Int J Syst Bacteriol. 1998;48(Pt 2):549-63.

67. Goris J, Dejonghe W, Falsen E, De Clerck E, Geeraerts B, Willems A, Top EM, Vandamme P, De Vos P. Diversity of transconjugants that acquired plasmid pJP4 or pEMT1 after inoculation of a donor strain in the A- and B-horizon of an agricultural soil and description of Burkholderia hospita sp. nov. and Burkholderia terricola sp. nov. Syst Appl Microbiol. 2002;25(3):340-52.

68. Brämer CO, Vandamme P, Da SL, Gomez JG, Steinbüchel A. Burkholderia sacchari sp. nov., a polyhydroxyalkanoate-accumulating bacterium isolated from soil of a sugar-cane plantation in Brazil. Int J Syst Evol Microbiol. 2001; 51(Pt 5):1709-13.

69. Zolg W, Ottow JC. Pseudomonas glathei sp. nov., a new nitrogen-scavening rod isolated from acid lateritic relicts in Germany. J Comp Neurol. 1975; 164(1):287-99.

70. Vandamme P, Mahenthiralingam E, Holmes B, Coenye T, Hoste B, De Vos P, Henry D, Speert DP. Identification and population structure of Burkholderia stabilis sp. nov. (formerly Burkholderia cepacia genomovar IV). J Clin Microbiol. 2000:38(3):1042-7.

71. Urakami T, Itoyoshida C, Araki H, Kijima T, Suzuki Kl, Komagata K. Transfer of Pseudomonas plantarii and Pseudomonas glumae to Burkholderia as Burkholderia spp. and description of Burkholderia vandii sp. nov. Int J Syst Bacteriol. 1994:44(2):235-45.

72. Holden MT, Seth-Smith HM, Crossman LC, Sebaihia M, Bentley SD, CerdenoTarraga AM, Thomson NR, Bason N, Quail MA, Sharp S, et al. The genome of Burkholderia cenocepacia J2315, an epidemic pathogen of cystic fibrosis patients. J Bacteriol. 2009;191(1):261-77.

73. Vandamme P, Holmes B, Vancanneyt M, Coenye T, Hoste B, Coopman R, Revets H, Lauwers S, Gillis M, Kersters K, et al. Occurrence of multiple genomovars of Burkholderia cepacia in cystic fibrosis patients and proposal of Burkholderia multivorans sp. nov. Int J Syst Bacteriol. 1997;47(4):1188-200.

Ready to submit your research? Choose BMC and benefit from:

- fast, convenient online submission

- thorough peer review by experienced researchers in your field

- rapid publication on acceptance

- support for research data, including large and complex data types

- gold Open Access which fosters wider collaboration and increased citations

- maximum visibility for your research: over $100 \mathrm{M}$ website views per year

At $\mathrm{BMC}$, research is always in progress.

Learn more biomedcentral.com/submissions 